\title{
ANÁLISE DE SUPERSATURAÇÃO, INTENSIDADE DE VIBRAÇÃO E TEMPO DE OPERAÇÃO NA PRODUÇÃO DE CRISTAIS DE ÁCIDO CÍTRICO E SACAROSE EM CRISTALIZADOR DE LEITO VIBRADO
}

\author{
D. B. QUINTINO ${ }^{1}$, R. A. MALAGONI ${ }^{1 *}$ \\ ${ }^{1}$ Universidade Federal de Uberlândia, Faculdade de Engenharia Química \\ *e-mail: malagoni@feq.ufu.br
}

\begin{abstract}
RESUMO
A sacarose e o ácido cítrico são amplamente estudados em pesquisas, devido à sua ampla utilização em produtos alimentícios, na indústria química e na produção de fármacos. Através da análise canônica dos resultados provenientes de um planejamento de experimentos, este estudo analisou a influência do índice de supersaturação, da vibração do meio cristalizante e do tempo de operação no processo de cristalização do ácido cítrico e da sacarose, por meio das respostas produtividade e do tamanho médio final do cristal. Para o planejamento de experimentos, observou-se as diferenças em granulometria e região de metaestabilidade entre a sacarose e o ácido cítrico fazendo-se adaptação ao procedimento experimental para os ensaios de sacarose, em comparação ao procedimento adotado em Teixeira et al (2012) na cristalização do ácido cítrico. Os resultados para ambos os compostos demonstraram-se semelhantes ao exigirem altos graus de supersaturação e baixos valores para o adimensional de vibração, fazendo somente distinção ao tempo de operação, sendo este último maior na cristalização de ácido cítrico. Os altos índices alcançados para dimensão do cristal e rendimento, associado à confirmação estatística, demonstraram a eficiência do equipamento no processo de separação sólido líquido.
\end{abstract}

\section{INTRODUÇÃO}

\subsection{A sacarose}

No Brasil, a cana-de-açúcar (Saccharum spp.) é cultivada desde o século XVI e apresenta atualmente um forte papel na economia brasileira. A cana-de-açúcar brasileira destaca-se por ser alterada geneticamente, e possuir baixos teores de fibra e altos valores de açúcar (Caputo et al., 2007). O Brasil, maior exportador de açúcar da atual década, em 2013, foi detentor de 50\% da exportação mundial conforme Unica (2013). A sacarose $\left(\mathrm{C}_{12} \mathrm{H}_{22} \mathrm{O}_{11}\right)$ é amplamente pesquisa em estudos voltados para o ramo de alimentos por desempenhara um importante papel como ingrediente e conservante em várias formulações e processos tecnológicos (QUINTAS et al., 2005).

\section{2 Ácido Cítrico}

O ácido cítrico é componente químico com formação baseada na fermentação de substratos de glucídios (BRAVI \& MAZZAROTTA, 1998).

Possui grande utilização na indústria de alimentos, bebidas e fármacos, além de ser empregado em uma vasta quantidade de processos industriais (NOTHENBERG, 1983). Na indústria alimentícia, é utilizado em larga escala como acidulante por apresentar sabor agradável, níveis mínimos de toxicidade e alta solubilidade (SOCCOL et $a l ., 2003)$. $\mathrm{Na}$ indústria química e de 
fármacos, possui aplicação na produção de detergentes, shampoos, cosméticos e produtos de limpeza em geral (SANTOS, 2008).

\subsection{Cristalização em leito vibrado}

$O$ processo de cristalização se desenvolve por dois mecanismos simultâneos principais: a nucleação e o crescimento dos cristais, que juntos com fenômenos secundários vão determinar a distribuição granulométrica dos cristais, caracterizada por um tamanho médio e sua dispersão (Derenzo, 2003). Em termos de Engenharia Química, a operação unitária da cristalização é um processo de transferência de massa e calor simultâneos, com forte dependência da dinâmica do fluído partícula (MYERSON, 2002; TEIXEIRA, 2011).

Devido ao seu simples modo de operação e controle de variáveis, sua fácil construção, por possuir baixo índice de manutenção e possibilitar variadas quantidades de bateladas, os cristalizadores batelada têm sido amplamente utilizados em pesquisas atualmente (OUIAZZANE et al., 2007).

O objetivo deste trabalho foi comparar a influência das principais variáveis operacionais no processo de cristalização de ácido cítrico e sacarose em um cristalizador de leito vibrado, mediante um planejamento de experimentos para os dois compostos, analisando sua configuração de produção máxima, associada ao maior tamanho de cristal. Os dados referentes ao ácido cítrico, foram obtidos de Teixeira et al (2012).

\section{MATERIAL E MÉTODOS}

Os experimentos de cristalização em leito vibrado foram realizados no Laboratório de Cristalização da Universidade Federal de Uberlândia. A Figura 1 apresenta os equipamentos utilizados nos ensaios de cristalização de ácido cítrico e sacarose, na qual temos: A - Cristalizador batelada;
B - Banho termostatizado (Tecnal, TE-184); C - Motor elétrico (WEG, 0,75CV); D Inversor de frequência (WEG, CFW-08), E Estufa (Medicate, modelo MD 1.3).

Figura 1 - Principais equipamentos utilizados nos experimentos de cristalização.

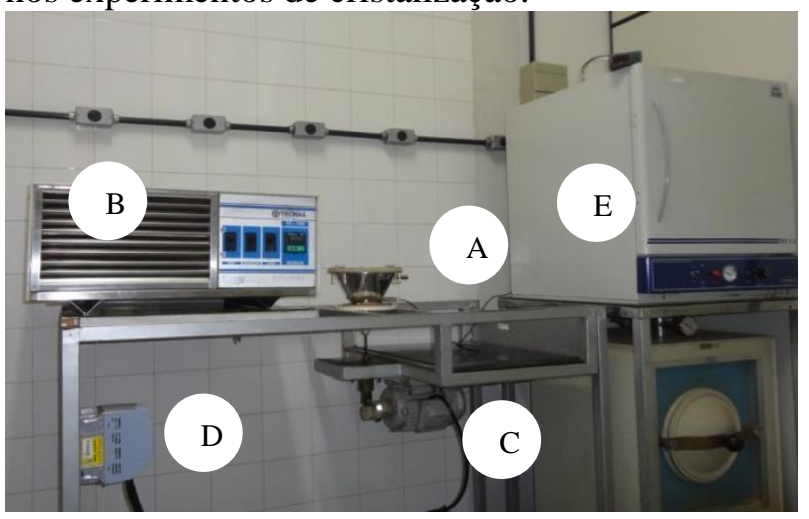

Fonte: Quintino (2014).

\subsection{Cristalizador Batelada de leito vibrado}

O cristalizador de leito vibrado foi confeccionado em aço inoxidável e é encamisado internamente. Em sua parte inferior, o equipamento foi vedado por uma membrana flexível de borracha sintética, própria para o processamento de alimentos. $\mathrm{O}$ equipamento possui formato tronco-cônico e sua seção cônica tem angulação de $65^{\circ} \mathrm{com}$ a horizontal, seu diâmetro superior e inferior possuem respectivamente $0,155 \mathrm{~m}$ e $0,064 \mathrm{~m}$, conforme demonstra a Figura 1.

Figura 2 - Cristalizador batelada de leito vibrado.

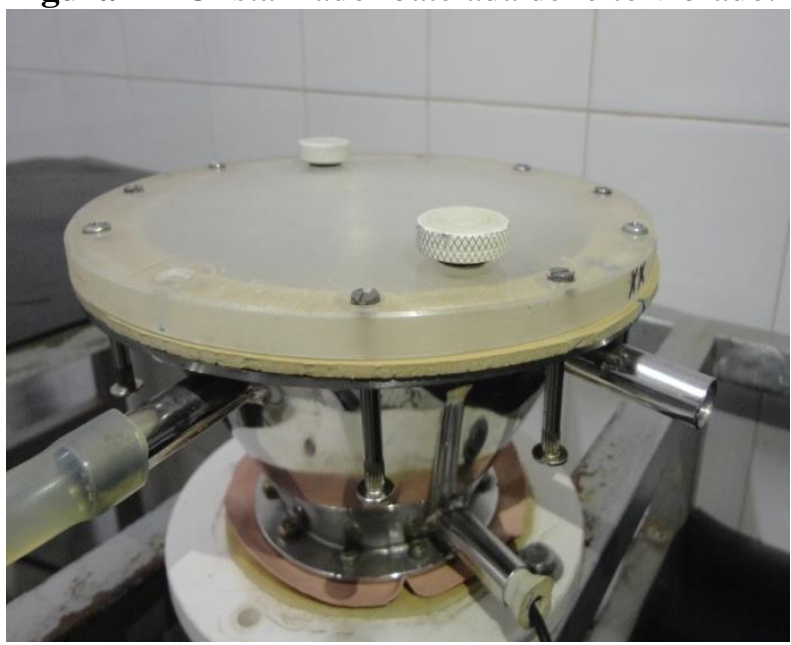


Fonte: Quintino, 2014.

A energia transmitida ao leito foi gerada pelo motor de corrente alternada (WEG, 0,75 $\mathrm{CV}$ ), este teve sua velocidade controlada através do inversor de frequência (WEG, modelo CFW-08). O sistema de agitação da solução do cristalizador é realizado por meio de pratos perfurados fixados ao eixo central do equipamento.

Na Figura 3, nota-se que o sistema foi constituído de dois discos perfurados feitos de aço inoxidável, uma presilha e três anéis de alumínio. $\mathrm{O}$ disco de maior dimensão possui 0,08 m de diâmetro, e é perfurado por 40 furos circulares de $5,0 \times 10^{-3} \mathrm{~m}$ cada. Já o disco de menor dimensão, possui $0,06 \mathrm{~m}$ de diâmetro, e possui 16 furos circulares de $5,0 \times 10^{-3} \mathrm{~m}$. Os anéis que promoveram a separação dos pratos foram confeccionados em alumínio, possuindo diâmetro interno de $13 \times 10^{-3} \mathrm{~m}$.

Figura 3 - Sistema de vibração da solução.

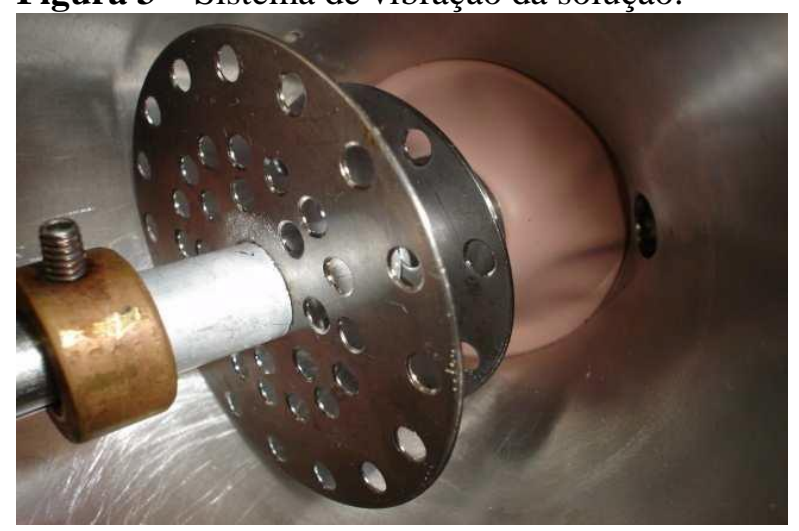

Fonte: Teixeira, 2011.

\subsection{Variáveis Utilizadas e Respostas Analisadas}

Três variáveis independentes foram analisadas a fim de determinar a produtividade e o tamanho médio do cristal para ambos os compostos, a saber: coeficiente de supersaturação, número adimensional de vibração e tempo de operação.

O coeficiente de supersaturação $(S)$, foi obtido através da Equação 1, na qual $C$ representa a concentração do sistema na temperatura de operação, e $C^{*}$ a concentração de saturação na temperatura do sistema.

$$
S=\frac{C}{C^{*}}
$$

O número adimensional de vibração foi calculado através da Equação 2, em que $A$ e $\omega$ são respectivamente, a amplitude de vibração (m) e a frequência angular de vibração $\left(\mathrm{s}^{-1}\right)$ do agente excitador externo que promove a vibração no leito, e $g$ representa a aceleração da gravidade no local.

$\Gamma=\frac{A \omega^{2}}{g}$

O tempo de operação variou entre 30 minutos e duas horas. $\mathrm{O}$ cálculo da produtividade cristalina foi realizado através da Equação 3, na qual $m_{c}$ representa a massa (g) de cristais obtida ao final do processo de cristalização e $m_{s}$ a massa $(\mathrm{g})$ de sementes introduzidas no cristalizador no início do processo de cristalização.

$$
y(\%)=\left(\frac{m_{c}}{m_{s}}-1\right) .100
$$

Para a outra resposta analisadas, o Diâmetro de Sauter, utilizou-se a equação 4, nesta, relacionado a fração mássica de sementes retidas $\left(\Delta x_{i}\right)$ com o diâmetro médio da porção retida $\left(D_{i}\right)$ em $\mathrm{mm}$.

$$
\bar{D}_{s t}=\frac{1}{\sum_{i=1}^{p} \frac{\Delta x_{i}}{D_{i}}}
$$

\subsection{Procedimento Experimental de Cristalização}

Uma solução supersaturada de acordo com o Planejamento de Experimentos foi preparada em banho-maria, com dados de solubilidade de sacarose e ácido cítrico obtidos respectivamente em Oetterer (2006), e 
Oliveira (2009). Após a solubilização da amostra, esta foi transferia ao cristalizador, este previamente montado e aquecido. Realizada a transferência, a temperatura de solução foi reduzida gradativamente à temperatura de operação.

Estabilizado o sistema termicamente, fez-se a adição das sementes e iniciou-se a contagem do tempo de cristalização. $\mathrm{Na}$ cristalização de sacarose, ao findar o processo, todo o material foi despejado sobre uma peneira de malha Tyler $24(0,707 \mathrm{~mm})$ previamente aquecida à temperatura de $333,2 \mathrm{~K}$, realizando assim a separação dos cristais formados da solução. Concomitantemente, os cristais foram lavados em solução alcoólica de $75 \%$ e $96 \%$, conforme analisado por Pereira (1997). Após separação, os cristais foram secos ao permanecer na estufa por $48 \mathrm{~h}$ a temperatura de $333,2 \mathrm{~K}$.

No processo envolvendo cristalização de ácido cítrico, devido a granulometria do material, a separação dos cristais da solução da solução mãe foi realizada utilizando-se um kitasato (Vidrolabor) de $500 \mathrm{ml}$, um funil de Buchner em cerâmica, papel de filtro quantitativo de $12,5 \mathrm{~cm}$ com poros de $14 \mu \mathrm{m}$ e uma bomba de vácuo (Primar, modelo 101). Outras diferenças adotadas no procedimento experimental, devido às diferenças em granulometria e região de metaestabilidade, são mencionadas na Tabela 1.

Tabela 1 - Procedimentos adotados devido às diferenças de região de metaestabilidade e granulometria.

\begin{tabular}{|c|c|c|}
\hline Operação & Sacarose & $\begin{array}{l}\text { Ácido } \\
\text { Cítrico }\end{array}$ \\
\hline $\begin{array}{l}\text { População de } \\
\text { sementes }\end{array}$ & $\begin{array}{l}0,042 \mathrm{~g} / \mathrm{mL} \\
\text { de solução }\end{array}$ & $\begin{array}{l}0,244 \mathrm{~g} / \mathrm{mL} \\
\text { de solução }\end{array}$ \\
\hline $\begin{array}{l}\text { Temperatura de } \\
\text { operação }\end{array}$ & $70^{\circ} \mathrm{C}$ & $55^{\circ} \mathrm{C}$ \\
\hline $\begin{array}{l}\text { Tempo de } \\
\text { secagem }\end{array}$ & $48 \mathrm{~h}$ & $24 \mathrm{~h}$ \\
\hline
\end{tabular}

\subsection{Planejamento de experimentos}

Um planejamento composto central (PCC) de 17 experimentos foi realizado para ambos os compostos.

O PCC foi constituído de seis experimentos nos pontos axiais, isto é, pontos nos quais uma das variáveis analisadas operou em condição extrema, e por três experimentos em condições operacionais em valores intermediários e idênticos, conhecidos como pontos centrais, correspondendo aos três últimos experimentos 15,16 e 17 .

Levando em consideração as regiões de metaestabilidade de cada um dos compostos, bem como a fluidização da solução devido às características das sementes introduzidas na solução, o planejamento de experimentos para a sacarose e para o ácido cítrico é apresentado na Tabela 2.

As Tabelas 3 e 4 abordam as variáveis codificadas e seus valores que para a sacarose e para o ácido cítrico respectivamente.

Tabela 2 - Planejamento Composto Central (PCC).

\begin{tabular}{cccc}
\hline Exp. & $x_{1}$ & $x_{2}$ & $x_{3}$ \\
\hline 1 & $-1,000$ & $-1,000$ & $-1,000$ \\
2 & $+1,000$ & $-1,000$ & $-1,000$ \\
3 & $-1,000$ & $+1,000$ & $-1,000$ \\
4 & $+1,000$ & $+1,000$ & $-1,000$ \\
5 & $-1,000$ & $-1,000$ & $+1,000$ \\
6 & $+1,000$ & $-1,000$ & $+1,000$ \\
7 & $-1,000$ & $+1,000$ & $+1,000$ \\
8 & $+1,000$ & $+1,000$ & $+1,000$ \\
9 & $-1,353$ & 0,000 & 0,000 \\
10 & $+1,353$ & 0,000 & 0,000 \\
11 & 0,000 & $-1,353$ & 0,000 \\
12 & 0,000 & $+1,353$ & 0,000 \\
13 & 0,000 & 0,000 & $-1,353$ \\
14 & 0,000 & 0,000 & $+1,353$ \\
15 & 0,000 & 0,000 & 0,000 \\
16 & 0,000 & 0,000 & 0,000 \\
17 & 0,000 & 0,000 & 0,000 \\
\hline
\end{tabular}


Tabela 3 - Níveis codificados do PCC para sacarose.

\begin{tabular}{llllll}
\hline $\begin{array}{l}\text { Var./ } \\
\text { Cod. }\end{array}$ & $-1,353$ & $-1,000$ & 0,000 & $+1,000$ & $+1,353$ \\
\hline$S / x_{1}$ & 1,020 & 1,050 & 1,110 & 1,170 & 1,200 \\
$\Gamma / x_{2}$ & 0,333 & 0,433 & 0,763 & 1,093 & 1,193 \\
$t / x_{3}$ & 0,500 & 0,696 & 1,250 & 1,804 & 2,000 \\
\hline
\end{tabular}

Tabela 4 - Níveis codificados do PCC para o ácido cítrico.

\begin{tabular}{cccccc}
\hline $\begin{array}{l}\text { Var./ } \\
\text { Cod. }\end{array}$ & $-1,353$ & $-1,000$ & 0,000 & $+1,000$ & $+1,353$ \\
\hline$S / x_{1}$ & 1,130 & 1,150 & 1,200 & 1,250 & 1,270 \\
$\Gamma / x_{2}$ & 0,440 & 0,530 & 0,780 & 1,030 & 1,110 \\
$t / x_{3}$ & 1,320 & 1,500 & 2,000 & 2,500 & 2,670 \\
\hline
\end{tabular}

\section{RESULTADOS E DISCUSSÃO}

Os valores obtidos de produtividade e diâmetro médio de Sauter nos experimentos do PCC, para ambos os produtos, são apresentados na Tabela 5.

Tabela 5 - Resultados experimentais na cristalização da sacarose e ácido cítrico.

\begin{tabular}{ccccc}
\hline & \multicolumn{2}{c}{ Sacarose } & \multicolumn{2}{c}{ Ácido Cítrico } \\
\hline Exp. & $y(\%)$ & $\bar{D}_{s t}$ & $y(\%)$ & $\bar{D}_{s t}$ \\
\hline 1 & 61,16 & 1,112 & 97,98 & 1,016 \\
2 & 162,50 & 1,343 & 143,58 & 1,022 \\
3 & 94,75 & 1,234 & 134,60 & 1,105 \\
4 & 151,58 & 1,264 & 138,23 & 0,877 \\
5 & 93,51 & 1,056 & 145,22 & 1,062 \\
6 & 129,91 & 1,337 & 153,87 & 1,043 \\
7 & 122,33 & 1,109 & 139,98 & 1,033 \\
8 & 98,33 & 1,231 & 136,36 & 0,960 \\
9 & 117,85 & 1,124 & 125,77 & 0,908 \\
10 & 158,66 & 1,467 & 165,31 & 0,831 \\
11 & 77,25 & 1,296 & 119,05 & 0,998 \\
12 & 84,85 & 1,155 & 155,41 & 1,103 \\
13 & 98,33 & 1,104 & 118,99 & 0,990 \\
14 & 131,41 & 1,252 & 137,41 & 1,102 \\
15 & 92,66 & 1,131 & 132,09 & 0,934 \\
16 & 89,41 & 1,179 & 142,19 & 0,812 \\
17 & 91,50 & 1,141 & 145,55 & 0,855 \\
\hline
\end{tabular}

Observa-se na Tabela 7 que os maiores valores de produtividade foram nos ensaios com grau de supersaturação em níveis altos ou em seu valor máximo, como visto nos experimentos 2, 4 e 10 para sacarose, e nos experimentos 6 e 10 para o ácido cítrico. O valor médio de produtividade alcançado nos ensaios para sacarose e ácido cítrico foi respectivamente $109,18 \%$ e $137,15 \%$.

Nota-se também alta proximidade de produtividade e diâmetro médio de Sauter para os dois compostos nos pontos centrais (ensaios 15, 16 e 17), nos quais a condições de operação foram idênticas, indicando estabilidade operacional. No ensaio número 10, obteve-se alta resposta para ambos em compostos nas duas respostas analisadas, com exceção ao diâmetro de Sauter para o ácido cítrico, neste experimento utilizou-se alto índice de supersaturação, valores intermediários para $\mathrm{o}$ adimensional de vibração e tempo de cristalização.

A Figura 4 apresenta a superfície de resposta para a sacarose, na qual é observada a variação da supersaturação com a intensidade de vibração na resposta de diâmetro de Sauter.

Figura 4 - Superfície de resposta para sacarose em duas variáveis para o diâmetro médio de Sauter.

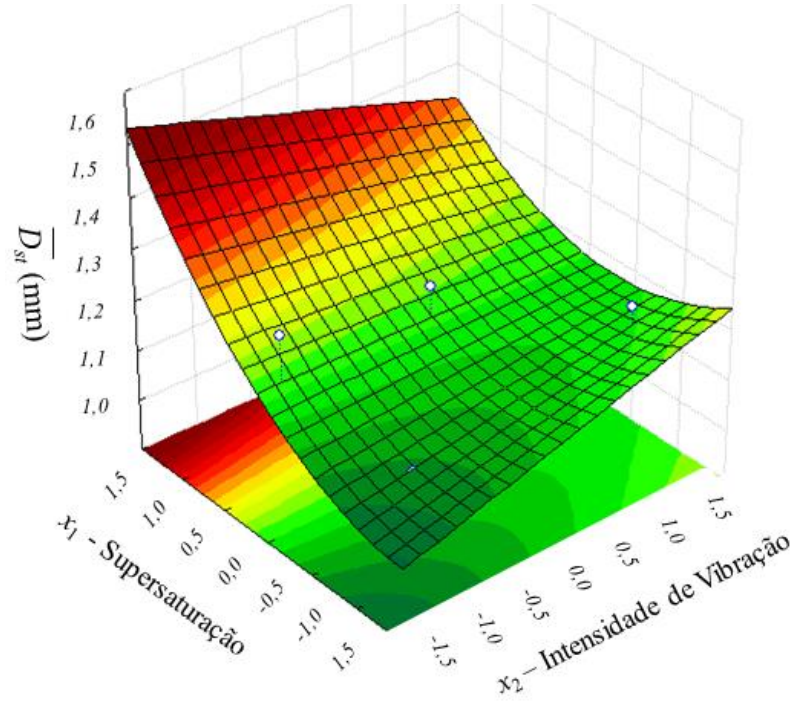


$\mathrm{Na}$ Figura 4, observa-se que cristais com maiores dimensões são obtidos para maiores valores de supersaturação e baixos valores de adimensionais de vibração. A Figura 5 apresenta a superfície de resposta obtida para produtividade cristalina de ácido cítrico, na variação do índice de supersaturação e da vibração. Observa-se que para um alto índice de supersaturação a variação da vibração do meio alterou pouco a produtividade, quando em comparação da variação da vibração para baixos índices de supersaturação.

Figura 5 - Resposta de produtividade cristalina do ácido cítrico na variação do adimensional de vibração e da supersaturação.

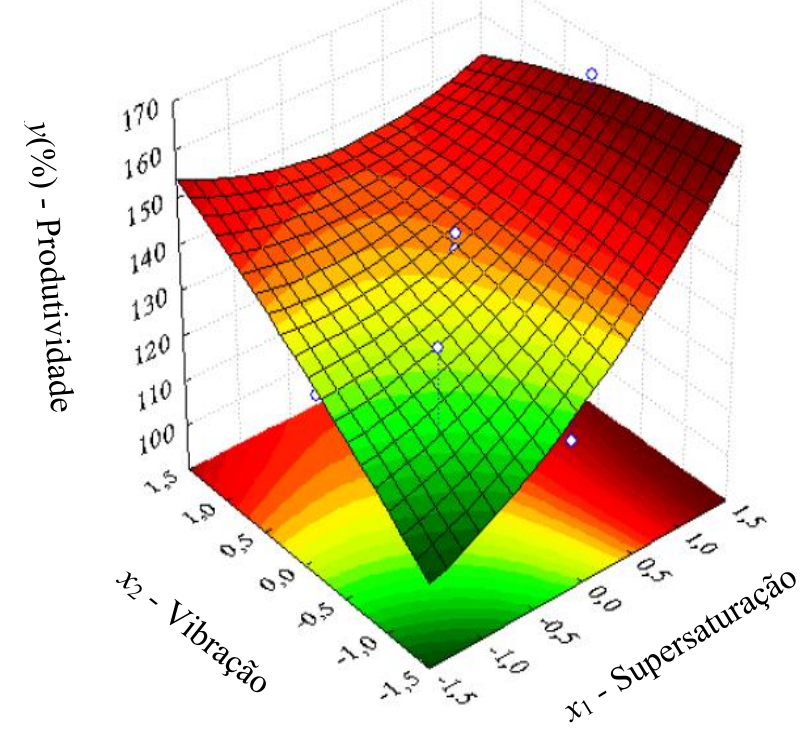

Calculou-se a condição operacional que maximizasse as respostas analisadas nos experimentos de cristalização, neste cálculo foi considerado como "desejabilidade" 1 a resposta máxima para produtividade $\mathrm{e}$ diâmetro de Sauter, valores inferiores de resposta mínima tiveram peso zero. Esta operação foi realizada após a análise canônica das equações dos modelos ajustados para as duas respostas para os dois compostos.
Os valores obtidos para sacarose foram: $S=1,20 ; \Gamma=0,514 ; t(\min )=82$. Para a cristalização de ácido cítrico, para a região de estudo, mediante a análise canônica, os valores que maximizam as duas respostas são: $S=1,27 ; \Gamma=1,05 ; t(\min )=104$.

Para ambos os produtos deste estudo, realizou-se teste em triplicata, nas condições operacionais apontadas pela análise estatística. Para a sacarose, o valor de produtividade e diâmetro médio de Sauter alcançados na condição de resposta máxima foi respectivamente, $163,12 \%$, e $1,481 \mathrm{~mm}$. Para o processo envolvendo o ácido cítrico, os valores obtidos para produtividade e diâmetro de Sauter foram respectivamente, $178,4 \%$ e $1,039 \mathrm{~mm}$.

Para sacarose, o modelo previu na condição de resposta máxima para a variável produtividade o valor de $162,64 \%$, para o diâmetro médio de Sauter, o valor predito foi de $1,466 \mathrm{~mm}$, resultando em uma diferença de menor que $2 \%$ entre o valor predito e o obtido para ambas as respostas neste composto.

Para o ácido cítrico, o valor de produtividade previsto pelo modelo foi $165 \%$, demonstrando que o obtido experimentalmente foi $8,3 \%$ superior. No diâmetro médio de Sauter, o valor médio na condição de resposta máxima, foi consideravelmente superior à média obtida no planejamento de experimentos, e semelhante ao previsto pelo modelo, o qual foi de $1,030 \mathrm{~mm}$.

\section{CONCLUSÃO}

Para a cristalização de sacarose em leito vibrado, pela análise estatística dos resultados experimentais, conclui-se na produção de resposta máxima para produtividade e diâmetro de Sauter, necessita-se de um alto índice de supersaturação associado a uma baixa vibração e tempo de operação superior a uma hora, um valor intermediário na faixa de estudo. 
Em condições de elevada intensidade de vibração em alto índice de supersaturação foi observado queda para ambas as respostas, fato que sugere o possível choque entre os cristais ocasionando quebra, diminuindo consequentemente $\mathrm{o}$ tamanho final dos mesmos. A respeito desta possível quebra de cristais, a análise microscópica destes revelaria a ocorrência do possível choque e quebra cristalino.

Semelhantemente à sacarose, na análise da cristalização de ácido cítrico, a superfície de resposta mostrou uma maior produtividade quando se trabalha com altos graus de supersaturação e baixos valores para o adimensional de vibração na faixa estudada. $\mathrm{Na}$ condição de resposta máxima, o tempo de cristalização obtido foi mais alto do que o obtido pela sacarose. A baixa intensidade de vibração favoreceu a transferência de massa para a superfície cristalina mediante a redução da camada limite em torno do cristal.

Nos ensaios em triplicata, na condição de reposta máxima obtidos estatisticamente, os valores resultantes demonstraram-se superiores aos alcançados em todo planejamento de experimentos, demonstrando a eficiência do equipamento no processo de separação associado reprodutibilidade dos dados, devido aos altos índices alcançados nas respostas, estas corroboradas estatisticamente, e aos valores similares dos pontos centrais.

\section{NOMENCLATURA}

\begin{tabular}{|c|l|r|}
\hline$A$ & Amplitude de vibração & {$[\mathrm{m}]$} \\
\hline$C$ & $\begin{array}{l}\text { Concentração do } \\
\text { sistema na temperatura } \\
\text { de operação }\end{array}$ & $\begin{array}{r}{[\mathrm{kg} / \mathrm{kg} \mathrm{de}} \\
\text { água }]\end{array}$ \\
\hline$C^{*}$ & $\begin{array}{l}\text { Concentração de } \\
\text { saturação na } \\
\text { temperatura do sistema }\end{array}$ & $\begin{array}{r}{[\mathrm{kg} / \mathrm{kg} \mathrm{de}} \\
\text { água }]\end{array}$ \\
\hline$D_{\mathrm{i}}$ & $\begin{array}{l}\text { Diâmetro médio da } \\
\text { porção retida }\end{array}$ & {$[\mathrm{mm}]$} \\
\hline$g$ & $\begin{array}{l}\text { Aceleração da } \\
\text { gravidade }\end{array}$ & {$\left[\mathrm{m} / \mathrm{s}^{2}\right]$} \\
\hline
\end{tabular}

\begin{tabular}{|c|l|r|}
\hline$m_{c}$ & Massa de cristais & {$[\mathrm{g}]$} \\
\hline$m_{\mathrm{s}}$ & Massa de sementes & {$[\mathrm{g}]$} \\
\hline PCC & $\begin{array}{l}\text { Planejamento } \\
\text { Composto Central }\end{array}$ & {$[-]$} \\
\hline$S$ & $\begin{array}{l}\text { Coeficiente de } \\
\text { supersaturação }\end{array}$ & {$[$ [-] } \\
\hline$x_{i}$ & $\begin{array}{l}\text { Variável codificada } \\
\text { analisada }\end{array}$ \\
\hline$y$ & $\begin{array}{l}\text { Produtividade de } \\
\text { cristais }\end{array}$ & {$\left[\mathrm{rpm}^{2}\right.$} \\
\hline$v$ & $\begin{array}{l}\text { velocidade do } \\
\text { excêntrico }\end{array}$ & {$[-]$} \\
\hline$\Gamma$ & $\begin{array}{l}\text { adimensional de } \\
\text { vibração }\end{array}$ & $\begin{array}{l}-1 \\
\text { frequência angular de } \\
\text { vibração }\end{array}$ \\
\hline$\omega$
\end{tabular}

\section{REFERÊNCIAS}

BRAVI, M.; MAZZAROTTA, B. Size dependency of citric acid monohydrate growth kinetics. Chemical Engineering Journal, v.70, p.203-207, 1998.

CAPUTO, M. M.; SILVA, M. A.; BEAUCLAIR, E. G. F.; GAVA, G. J. C. Acúmulo de sacarose, produtividade e florescimento de cana-de-açúcar sob reguladores vegetais. Interciencia, vol.32, p.834-840, 2007.

DERENZO, S. Cristalização de ácido adípico em diferentes solventes. 2003. Tese (Doutorado em Engenharia Química) Universidade Federal de São Carlos, São Carlos, 2003.

MYERSON, A. S. Handbook of Industrial Crystallization. Boston: ButterworthHeinemann, 2002.

NOTHENBERG, M. Novos mercados reativam a produção. Química e derivados, v.205, p.38-43, 1983. 
OETTERER, M.; SARMENTO, R. d'D. M. A.; SPOTO, M. H. F. Fundamentos de Ciência e Tecnologia de Alimentos, Barueri: Manole, 2006

OLIVEIRA, M. L. N. Estudo experimental das solubilidades de alguns ácidos em misturas hidro alcoólicas. 2009. 340p. Tese (Doutorado em Engenharia Química) Universidade Federal de Uberlândia, Uberlândia, 2009.

OUIAZZANE, S.; MESSNAOUI, B.; ABDERAFI, S.; WOUTERS, J.; BOUNAHMIDI, T. Estimation of sucrose crystallization kinetics from batch crystallizer data. Journal of Crystal Growth, v.310, p.798-803, 2007.

QUINTAS, M.; BRANDÃO, T. R. S.; SILVA, C. L. M.; CUNHA, R. L. Rheology of supersaturated sucrose solutions. Journal of Food Engineering, v.77, p.844-852, 2005.

QUINTINO, D. B. Otimização operacional do processo de cristalização da sacarose em leito vibrado. 2014. 96p. Dissertação (Mestrado em Engenharia Química) Universidade Federal de Uberlândia, Uberlândia, 2014.

SANTOS, J. A. F. Avaliação dos resíduos dos processamentos de suco de laranja e de cerveja para obtenção de ácido cítrico por fermentação em estado sólido. 2008. 102f. Dissertação (Programa de Pós-graduação em Biotecnologia Industrial) - Universidade de São Paulo, Lorena, 2008.

TEIXEIRA, G. A., Estudo da Produtividade no Processo de Cristalização de Ácido Cítrico em Leito Vibrado. 2011. 144p. Dissertação (Mestrado em Engenharia Química) - Universidade Federal de Uberlândia, Uberlândia, 2011.
TEIXEIRA, G. A., VIEIRA, W. F., FINZER, J. R. D., MALAGONI, R. A., Citric acid crystallization process in dense phase using vibrated bed. Journal of Food Engineering, v.111, p.458-465, 2012.

UNICA. União da Indústria de Cana-deaçúcar. Sugarcane Industry in Brazil. São Paulo, SP, mai. de 2013. Disponível em: <http://www.unica.com.br/documentos.php $>$. Acessado em 10 de abril de 2015.

\section{AGRADECIMENTOS}

Este trabalho foi financeiramente apoiado pela CAPES. 